\title{
Gynecological tumors in Mulibrey nanism and role for RING finger protein TRIM37 in the pathogenesis of ovarian fibrothecomas
}

\author{
Susann Karlberg ${ }^{1,2}$, Marita Lipsanen-Nyman ${ }^{2}$, Heini Lassus ${ }^{1}$, Jukka Kallijärvi ${ }^{3,4,5}$, \\ Anna-Elina Lehesjoki ${ }^{3,4,5}$ and Ralf Butzow ${ }^{1,6}$ \\ ${ }^{1}$ Department of Obstetrics and Gynecology, Helsinki University Central Hospital, Biomedicum Helsinki, \\ Helsinki, Finland; ${ }^{2}$ Children's Hospital, University of Helsinki, Helsinki, Finland; ${ }^{3}$ Folkhälsan Institute of \\ Genetics, Helsinki, Finland; ${ }^{4}$ Department of Medical Genetics, University of Helsinki, Helsinki, Finland; \\ ${ }^{5}$ Neuroscience Center, University of Helsinki, Helsinki, Finland and ${ }^{6}$ Department of Pathology, University of \\ Helsinki, Helsinki, Finland
}

\begin{abstract}
Mulibrey nanism is an autosomal recessive growth disorder caused by mutations in the TRIM37 gene encoding a protein of unknown function. More than half of female patients with Mulibrey nanism develop benign mesenchymal tumors of ovarian sex cord-stromal origin. In this work, we characterize the gynecological tumors of female patients with Mulibrey nanism in detail. In addition to tumors of the fibrothecoma group, $18 \%$ (4/22) of the patients were observed with epithelial neoplasias, including 2 ovarian adenofibromas, 1 ovarian poorly differentiated adenocarcinoma and 1 endometrial adenocarcinoma. To investigate the possible involvement of TRIM37 alterations in the pathogenesis of sporadic fibrothecomas, we analyzed the TRIM37 cDNA for mutations and alternatively spliced transcripts and TRIM37 expression in fibrothecomas of women without Mulibrey nanism. No mutations in the open-reading frame of TRIM37 were detected. Two alternatively spliced variants were found, one lacking exon 23 and one exon 2. TRIM37del2 was also found in normal ovary but in a proportion of sporadic fibrothecomas, the TRIM37del2:TRIM37 ratio was increased. In normal ovary, TRIM37 was localized in the cytoplasm of stromal cells, especially theca cells surrounding developing follicles. TRIM37 transcript was found in all sporadic fibrothecomas examined, but $80 \%(20 / 25)$ of the tumors showed reduced or absent expression of TRIM37 protein. Allelic loss at the TRIM37 locus (17q22-23) was observed in $6 \%$ of sporadic fibrothecomas. Nearly half of the sporadic fibrothecomas showed evidence of CpG promoter methylation, suggesting promoter downregulation as one mechanism of reduced TRIM37 expression. In conclusion, inherited biallelic inactivation of TRIM37 (Mulibrey nanism) predisposes to both mesenchymal and epithelial ovarian tumors and dysregulation of TRIM37 may also be involved in the pathogenesis of sporadic fibrothecomas.
\end{abstract}

Modern Pathology (2009) 22, 570-578; doi:10.1038/modpathol.2009.13; published online 27 February 2009

Keywords: ovarian tumor; fibrothecoma; hereditary predisposition; hereditary disorder; TRIM37; Mulibrey nanism

Mulibrey nanism is an autosomal recessive inherited disorder characterized by prenatal-onset growth failure, distinct craniofacial features, perimyocardial heart disease, insulin resistance and a mainly unaffected psychomotor development. ${ }^{1-4}$ This disorder is enriched in Finland where 88 of the approximately 130 known patients have been

Correspondence: Dr R Butzow, MD, PhD, Department of Pathology, University of Helsinki, P.O. Box 21, FIN 00014, Helsinki, Finland.

E-mail: ralf.butzow@hus.fi

Received 11 December 2008; revised 2 February 2009; accepted 3 February 2009; published online 27 February 2009 diagnosed. Mulibrey nanism is caused by mutations in the TRIM37 gene on chromosome $17 \mathrm{q} 22-23 .^{5-7}$ It codes for a protein belonging to the TRIM (TRIpartite Motif; previously designated RBCC for RING-Bbox-Coiled-coil) protein family, members of which are often involved in developmental regulation and oncogenesis. ${ }^{6}$ The wild-type TRIM37 protein localizes to peroxisomes in cultured human and rodent cells $^{8}$ and possesses ubiquitin E3 ligase activity. ${ }^{9}$ Fifteen disease-associated mutations in the TRIM37 gene have been published..$^{6,7,9-12}$ The recessive nature of the disorder and the fact that all but four of the disease-associated mutations are truncating suggest a loss-of-function modality underlying the 
pathogenesis of Mulibrey nanism. The physiological function of TRIM37 and the exact molecular mechanisms leading to Mulibrey nanism are unknown.

The development of reproductive organs seems to be unaffected in Mulibrey nanism. However, female patients with Mulibrey nanism are infertile and they all develop ovarian failure before the age of 30 years. ${ }^{13}$ We have previously reported that female patients with Mulibrey nanism are at a very high risk of developing ovarian sex cord-stromal tumors. ${ }^{13}$ However, these tumors have not been previously characterized in detail. In this study, we describe the clinical and pathological characteristics of all ovarian tumors associated with Mulibrey nanism. There are many examples of genes responsible for hereditary tumor syndromes being defective also in respective sporadic tumors. Therefore, we analyzed the TRIM37 gene and protein expression and looked for somatic mutations, loss of heterozygosity (LOH) and promoter methylation of the gene in sporadic fibrothecomas.

\section{Materials and methods}

\section{Patients with Mulibrey Nanism}

After characterization of Mulibrey nanism in the mid-1970s, a nationwide register of patients was established in Finland, and the follow-up of the patients has been performed and organized at the Children's Hospital, Helsinki University Central Hospital, mainly by one of the authors (ML-N). The complete records of 22 female patients $>20$ years of age were reviewed for ovarian and other gynecological tumors. Age at primary diagnosis or recurrences was recorded. The surgical reports were reviewed for tumor size, multifocality and bilaterality. The study was approved by the institutional ethical review board at the University of Helsinki, and all subjects gave informed consent.

\section{Tissue Samples}

All tissue samples were reviewed by a gynecological pathologist (RB) and the observed tumors were classified according to the criteria of WHO. ${ }^{14}$ The formalin-fixed and paraffin-embedded samples were obtained from the archives of the Department of Obstetrics and Gynecology, Helsinki University Central Hospital. The freshly frozen tumor specimens were collected at the time of surgery, snap frozen in liquid nitrogen and stored at $-80^{\circ} \mathrm{C}$. Normal ovary samples $(n=3)$ came from women ( $<35$ years) for whom oophorectomy was performed because of cervical carcinoma without neoadjuvant treatment. Normal tissue complementary DNAs (cDNAs) came from human multiple tissue cDNA panels (Human MTC panel I and Human MTC panel II; Clontech, Mountain View, CA, USA).
The study material was as follows: formalin-fixed paraffin-embedded tumors from patients with Mulibrey nanism $(n=12)$; freshly frozen samples $(n=15)$ from sporadic fibrothecomas for mutation analysis; mainly formalin-fixed paraffin-embedded samples with some freshly frozen samples for $\mathrm{LOH}$ analysis and methylation studies, 33 and 31 samples, respectively. Owing to limitations in the availability of tumor tissue, the study material used in different analyses was only partly overlapping.

Two tissue arrays were prepared and analyzed: (1) a macroarray consisting of core tissue biopsies ( $4 \mathrm{~mm}$ diameter each) from Mulibrey fibrothecomas $(n=12)$ and (2) a microarray from sporadic fibrothecomas $(n=25)$, which was constructed as described previously. ${ }^{15}$ Four core tissue biopsies were obtained from each specimen.

\section{Immunohistochemistry}

From the tumor tissue array blocks and normal ovary samples, $5-\mu \mathrm{m}$ sections were cut with a microtome. The primary antibody used for the Mulibrey fibrothecomas was anti-inhibin- $\alpha$ (MCA951S; Serotec, Oxford, UK; 1:80). For TRIM37 staining of sporadic fibrothecomas and normal ovary samples, an antigen affinity-purified fraction of rabbit antiserum $(60 \mu \mathrm{g} / \mu \mathrm{l})$ raised against a synthetic peptide (FPDGEQIGPEDLSFNTDENSGR) corresponding to the $\mathrm{C}$ terminus of the TRIM37 protein was used. ${ }^{8}$ Normal rabbit IgG (Vector Laboratories, Burlingame, CA, USA) was used as a negative control. TRIM37 staining was carried out as previously described, ${ }^{8}$ using Vectastain Elite kit (Vector Laboratories) according to the manufacturer's protocol. Immunostaining of inhibin- $\alpha$ was performed in a Dako TechMate 500 automated staining machine.

\section{Mutation Analysis of TRIM37 cDNA in Sporadic Fibrothecomas}

From freshly frozen samples of sporadic fibrothecomas $(n=15)$, messenger RNA was extracted using the Oligotex mRNA midi kit (Qiagen, Hilden, Germany). cDNA was synthesized using M-MLV reverse transcriptase (Promega, Madison, WI, USA) and random hexamers (Promega) according to the manufacturer's protocols. Seven overlapping fragments covering the whole open-reading frame of TRIM37 were amplified by polymerase chain reaction (PCR) using primers and conditions as before. ${ }^{6}$ The resulting PCR products were evaluated on $1 \%$ ethidium bromide-stained agarose gels, purified (PCR purification kit; Qiagen) and sequenced using the ABI PRISM Dye Terminator, the ABI PRISM dRhodamine cycle sequencing Kit (PerkinElmer, Foster City, CA, USA) or Big Dye Terminator (Applied Biosystems, Foster City, CA, USA) and run on an ABI Prism 310 Genetic Analyzer (Perkin Elmer). Both strands were sequenced and the 
obtained sequence information was edited, aligned and compared to TRIM37 cDNA sequence (GenBank NM 015294.1) using Sequencher 3.1 (Genes Codes Corporation).

\section{LOH at 17q11-25 in Sporadic Fibrothecomas}

LOH was assessed at 17q11-25 and particularly around the TRIM37 locus at 17q22-23 in 33 sporadic fibrothecomas of which sufficient material was available. Tumor and respective normal DNA was extracted according to standard protocols. A set of 10 highly polymorphic microsatellite markers (D17S1824, D17S1872, D17S1861, D17S1607, D17S1606, D17S1853, D17S1604, D17S948, D17S1806 and D17S1830) were used to determine LOH status; 3 markers situated clearly proximal to TRIM37, 2 distal to TRIM37 and 5 close to TRIM37 (17q22-23). Primer sequences and reaction conditions for nucleotide markers were obtained from the Genethon (http://ftp.genethon.fr) human linkage map. The genetic order of the markers was based on the Genethon map and the genomic location of the markers was confirmed by using the UCSC March 2006 assembly (NCBI Build 36.1) (Figure 3). The reactions were performed and the results analyzed as described. ${ }^{16,17}$ In ambiguous cases, the PCR, electrophoresis and scoring were repeated.

\section{TRIM37 Promoter Methylation Analysis in Sporadic Fibrothecomas}

Tumor DNA methylation was assessed in 31 sporadic fibrothecomas of which sufficient material was available, utilizing methylation-sensitive restriction enzyme HpaII (recognition site CCGG; New England Biolabs, Ipswich, MA, USA) and quantitative PCR. DNA (200 ng) was incubated at $37^{\circ} \mathrm{C}$ for $1 \mathrm{~h}$ with $25 \mathrm{U}$ of HpaII, $1.5 \mu \mathrm{l}$ of restriction enzyme buffer (New England Biolabs) and the appropriate amount of DNase-free water to give a final reaction volume of $15 \mu \mathrm{l}$. As individual sample controls we used undigested DNA and DNA digested with methylation-insensitive restriction enzyme MspI (recognition site CCGG; New England Biolabs). The CpGrich promoter region of TRIM37 was amplified with two different primer pairs that were designed by using Oligo 6.8 software (Molecular Biology Insights Inc., Cascade, CO, USA) together bracketing four CCGG sites. The primer sequences were as follows: TRIM37/1, F-5'-AGAGCCCCAAGCTCAGG-3', R-5'-CGCAAACACCAACCGTA-3' (a 221 bp product) and TRIM37/2, F-5'-CCGTCAGTTCCATAGGC-3', R-5'-GAGGCGCAGAAGTAGGG-3' (120 bp). Quantitative real-time PCR was carried out on an ABI Prism ( 7500 device. Each $20 \mu$ l reaction consisted of $0.8 \mu \mathrm{l}$ sense and antisense primer, $10 \mu \mathrm{l}$ SYBR Green Master Mix (Applied Biosystems), $7.4 \mu \mathrm{l}$ $\mathrm{dH}_{2} \mathrm{O}$ and $0.94 \mu \mathrm{l}$ DNA sample. PCR was carried out at $95^{\circ} \mathrm{C}$ for $10 \mathrm{~min}$, followed by 40 cycles at $95^{\circ} \mathrm{C}$ for $15 \mathrm{~s}$ and $60^{\circ} \mathrm{C}$ for $60 \mathrm{~s}$. Each run included genomic DNA from whole blood and water blanks as controls. A difference of one CT (cycle threshold value) between HpaII and MspI digested samples was used as a cutoff. In ambiguous cases, the samples were additionally evaluated on $2 \%$ agarose gels and stained with ethidium bromide.

\section{Results}

\section{Clinicopathologic Characteristics of Mulibrey Nanism-Associated Ovarian Tumors}

At the time of data analysis, the mean age of the patients was 35 years and four had died of complications related to Mulibrey nanism. One patient had died accidentally at the age of 41 with no autopsy performed. Altogether 12 of the 22 women (55\%) had been diagnosed with ovarian tumors (Table 1). The mean age at first tumor diagnosis was 29 years (range 16-52 years). Seven of the tumors were thecomas, four fibromas and one a cellular fibroma (Figure 1a-c). In two cases, in addition to fibromas, separate tumors composed of both stromal and epithelial cells with serous differentiation (serous adenofibroma, Figure 1d) were observed. Tumor size varied from 1 to $15 \mathrm{~cm}$ with a mean diameter of $7 \mathrm{~cm}$. Of the 12 tumors, 5 $(42 \%)$ were multifocal, and in 6 of the 12 cases $(50 \%)$ a tumor was present also in the contralateral ovary (Table 1). The smaller tumors were clearly demarked from the non-neoplastic ovarian tissue whereas in larger tumors the ovarian architecture was usually disturbed. Two patients had recurring fibrothecomas 18 and 17 years after the primary operation. One patient was diagnosed with poorly differentiated ovarian adenocarcinoma 11 years after unilateral oophorectomy performed because of thecoma. One patient presented with endometrial adenocarcinoma and a new thecoma 9 years after primary fibrothecoma. Of the 12 fibrothecomas, 9 $(75 \%)$ were positive for inhibin- $\alpha$ in immunohistochemical staining (Figure 1e).

\section{TRIM37 Protein in Normal Ovary and Sporadic Fibrothecomas}

In normal ovary, moderate to strong TRIM37 immunoreactivity was seen in the cytoplasm of theca cells surrounding developing follicles (theca interna). Other ovarian stromal cells showed weak TRIM37 positivity (Figure 1f). In $20 \%$ of the sporadic fibrothecomas (5/25) immunopositivity comparable to that seen in normal thecal cells was observed (Figure 1g). In $32 \%(8 / 25)$ of the tumors weak TRIM37 immunopositivity was observed (Figure 1h) and in $48 \%(12 / 25)$ of the cases the tumor cells were completely negative for TRIM37 (Figure 1i). In all the specimens vessel walls were 
Table 1 Ovarian tumors in 22 females with Mulibrey nanism

\begin{tabular}{|c|c|c|c|c|c|c|c|}
\hline No. & Year of birth & Tumor & $\begin{array}{c}\text { Age at diagnosis } \\
\text { (years) }\end{array}$ & Diameter $(\mathrm{cm})$ & Bilateral & Multifocal & Recurrence \\
\hline 1 & 1929 & $\mathrm{CF}$ & 52 & 8 & + & & \\
\hline 2 & $1936^{\mathrm{a}}$ & Thecoma & 36 & 15 & - & & 45 years; thecoma \\
\hline 3 & $1947^{\mathrm{b}}$ & & & & & & \\
\hline 4 & $1949^{\mathrm{c}}$ & Fibroma & 26 & 8 & + & + & \\
\hline 5 & 1949 & Fibroma + AF & 24 & 7 & + & + & 42 years; fibroma+AF \\
\hline 6 & 1950 & Fibroma+AF & 32 & 6 & + & + & 49 years; thecoma \\
\hline 7 & $1957^{\mathrm{d}}$ & Thecoma & 22 & 5 & + & + & \\
\hline 8 & 1959 & & & & & & \\
\hline 9 & $1960^{\mathrm{e}}$ & Thecoma & 22 & 1 & - & & \\
\hline 10 & 1964 & Thecoma & 35 & 5 & - & & \\
\hline 11 & 1964 & Thecoma & 35 & 1 & - & + & \\
\hline 12 & 1966 & & & & & & \\
\hline 13 & 1968 & & & & & & \\
\hline 14 & 1969 & & & & & & \\
\hline 15 & 1971 & & & & & & \\
\hline 16 & 1975 & & & & & & \\
\hline 17 & 1976 & Fibroma & 28 & 3 & + & & \\
\hline 18 & 1977 & & & & & & \\
\hline 19 & 1979 & & & & & & \\
\hline 20 & 1980 & Thecoma & 24 & 8 & - & & \\
\hline 21 & 1983 & Thecoma & 16 & 14 & - & & \\
\hline 22 & 1984 & & & & & & \\
\hline
\end{tabular}

$\mathrm{CF}$, cellular fibroma; AF, adenofibroma.

${ }^{\mathrm{a}}$ Death at 45 years (uterine adenocarcinoma).

${ }^{\mathrm{b}}$ Accidental death at 41 years (no autopsy).

${ }^{\mathrm{C}}$ Death at 39 years (sepsis).

${ }^{d}$ Death at 33 years (poorly differentiated ovarian adenocarcinoma).

${ }^{\mathrm{e}}$ Death at 22 years (cardiac insufficiency).

positive for TRIM37, serving as internal controls for the staining.

\section{TRIM37 Expression and Mutation Analysis in Sporadic Fibrothecomas}

TRIM37 cDNA was obtained from all 15 freshly frozen tumor specimens and sequenced from 7 overlapping amplicons. In one tumor, a base pair change from $\mathrm{C} \rightarrow \mathrm{T}$ at nucleotide 398 was revealed, resulting in a substitution of valine for alanine, but no other sequence alterations were observed. Amplification of two of the segments (primers $1 \mathrm{~F}$ with $1 \mathrm{R}$ and $7 \mathrm{~F}$ with $7 \mathrm{R}$, respectively ${ }^{6}$ ) constantly yielded two products, one of expected size representing a segment of the full-length cDNA and one smaller fragment. The unexpectedly small fragments were isolated and based on sequence data they originated from transcripts where exon 2 or exon 23 had been spliced off. TRIM37del2 was present in all tumors and the TRIM37del2:TRIM37 ratio varied across the tumor specimen (Figure 2). TRIM37del2 could be found also in normal ovary and to a lesser extent in several other tissues (Figure 2) indicating that TRIM37del2 is not tumor specific. However, in a proportion of sporadic fibrothecomas the TRIM37del2:TRIM37 ratio was increased (Figure 2).

\section{LOH at 17q11-25 in Sporadic Fibrothecomas}

Of 33 sporadic fibrothecomas, 2 (6\%) exhibited LOH of 3 markers (D17S1604, D17S948 and D17S1806) (Figure 3). A minimal common region of loss, which includes the locus for TRIM37, was defined between D17S1606 and D17S1830 (17q22-25).

\section{Methylation Analysis in Sporadic Fibrothecomas}

Basal promoter activity of TRIM37 has been mapped within $600 \mathrm{bp}$ upstream from the translation initiation site. ${ }^{18}$ This region contains eight CpG sites, of which we analyzed four in this work. Of the 31 sporadic fibrothecomas studied, 23 (74\%) were informative and in $11(48 \%)$ of the 23 cases either one or both of the amplified fragments showed evidence of hypermethylation in quantitative PCR using the set criteria (see Materials and methods section). TRIM37 immunohistochemical staining was available in 12 of the 23 informative cases in which methylation analysis was performed. Of these, TRIM37 immunostaining was weak or 


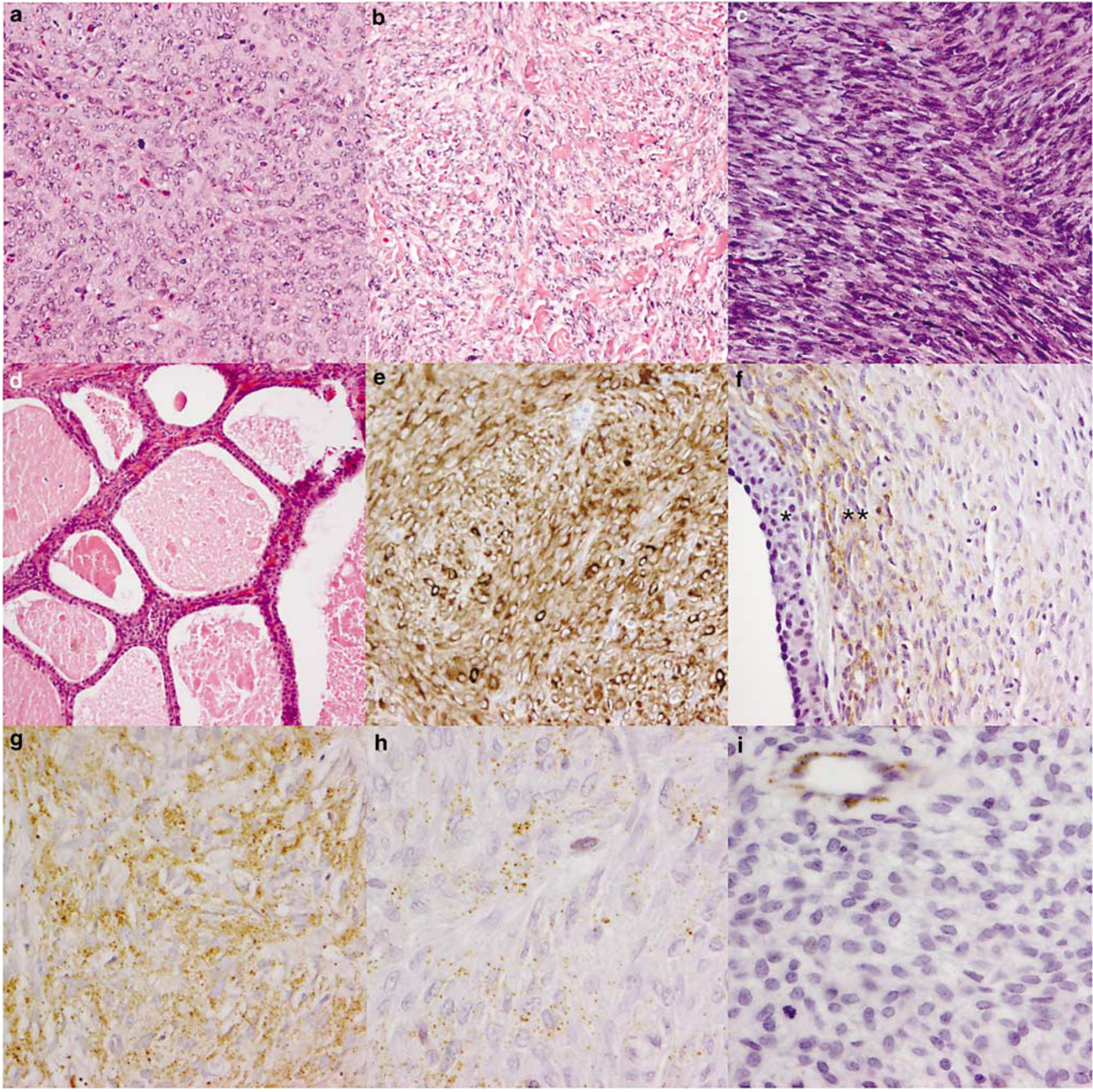

Figure 1 Examples of Mulibrey nanism-associated ovarian tumors (standard HE-staining) and immunohistochemical analysis of TRIM37 distribution in normal ovary and in sporadic fibrothecomas. (a) Mulibrey-associated thecoma; (b) Mulibrey-associated fibroma; (c) Mulibrey-associated cellular fibroma; (d) Mulibrey-associated adenofibroma; (e) inhibin- $\alpha$-positive Mulibrey-associated fibrothecoma; (f) normal ovary, ${ }^{*}$ : granulosa cells, ${ }^{* *}$ : thecal cells; (g) sporadic fibrothecoma with strong TRIM37 immunopositivity; (h) sporadic fibrothecoma with weak TRIM37 immunopositivity and (i) sporadic fibrothecoma completely negative for TRIM37, note positive staining of capillary wall cells. Original magnification $\times 200$.

negative in 9 tumors, and in 6 of these hypermethylation was present.

\section{Discussion}

During the follow-up of female patients with Mulibrey nanism, it was observed that in addition to premature ovarian failure many of the patients developed ovarian tumors. In our cohort 55\% (12/ 22) of the patients presented with ovarian tumors. Most of the tumors were morphologically and immunohistochemically indistinguishable from fibrothecomas, benign neoplasias originating from ovarian stromal or hormonally active thecal cells. Fibromas are composed of spindle-shaped cells 

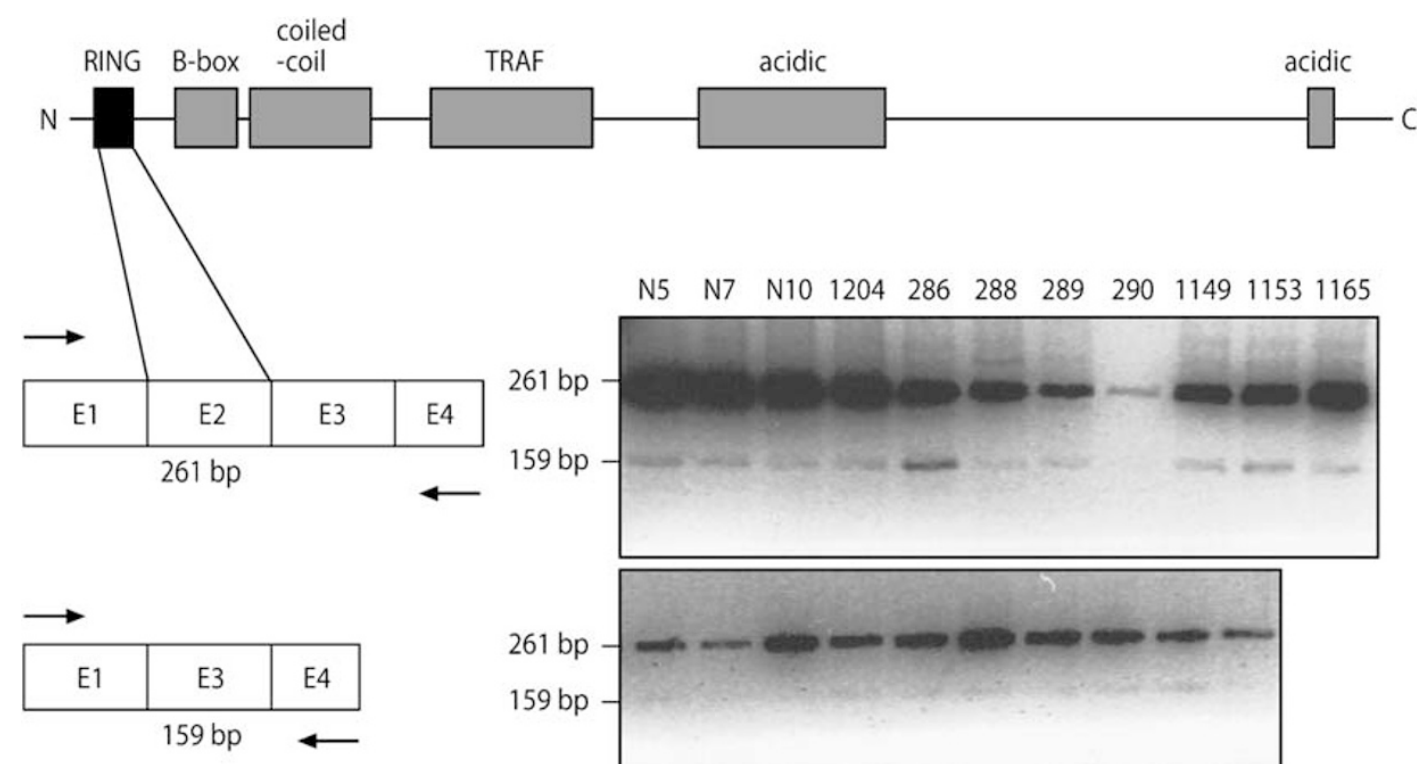

$\begin{array}{llllllllllll}\text { N5 } & \text { N7 } & \text { N10 } & 1204 & 286 & 288 & 289 & 290 & 1149 & 1153 & 1165\end{array}$
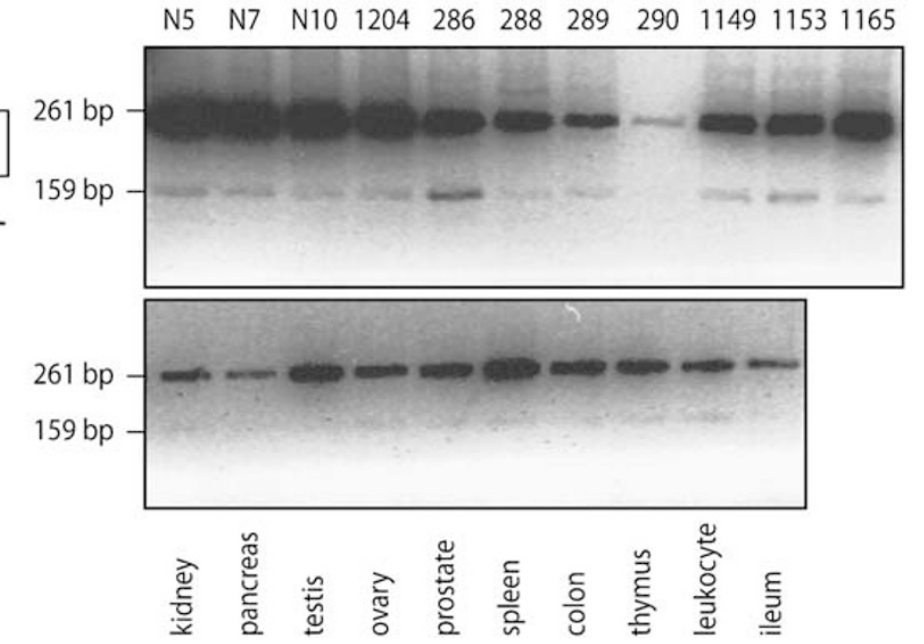

Figure 2 Schematic structure of TRIM37 and the TRIM37del2 splice variant; one fragment representing full-length cDNA (261 bp) and one fragment (159bp) lacking exon 2. TRIM37del2 in normal ovary: N5, N7, N10, in sporadic fibrothecomas: 1204, 286, 288, 289, 290, $1149,1153,1165$ and in various human tissues as indicated in the figure.

surrounded by collagen-rich matrix. They are not hormonally active, but their cells differ from 'normal' fibroblasts as indicated eg by inhibin- $\alpha$ expression. In thecomas the tumor cells have abundant and lipid-rich cytoplasm that relates to their steroidogenic activity. Thecomas may have fibroma-like areas and due to this overlap these tumors are often collectively referred to as fibrothecomas. These tumors are relatively uncommon (1-2\% of all ovarian tumors ${ }^{19}$ ) and usually postmenopausal (mean age 63 years ${ }^{20}$ ). In patients with Mulibrey nanism the tumors were early onset (mean age 29 years), a feature typical of an inherited tumor predisposition syndrome.

More than half of the Mulibrey nanism-associated fibrothecomas were multifocal or bilateral and in three cases the tumors recurred after primary ovary sparing surgery. In most cases the tumor was clearly demarked from the non-neoplastic ovarian tissue. These features together strongly suggest that the tumors represent true neoplasias and not hyperplasia of stromal cells or diffuse ovarian fibromatosis, a rare non-neoplastic disorder of unknown origin. ${ }^{19}$ Most Mulibrey nanism-associated fibrothecomas also expressed inhibin- $\alpha$, which is a typical feature of sporadic fibrothecomas.

In addition to fibrothecomas, $18 \%(4 / 22)$ of the patients with Mulibrey nanism had other gynecological tumors. Two had serous adenofibromas, which are benign tumors composed of a fibroma-like stromal component and glandular structures of ciliated epithelium resembling the fallopian tubes.
Despite their biphasic nature, these tumors are classified as epithelial. Their molecular background and relationship to malignant epithelial ovarian neoplasias is unknown. One patient had a poorly differentiated ovarian adenocarcinoma at the age of 33, suggesting that patients with Mulibrey nanism could be predisposed to ovarian carcinoma, as well. Removal of the ovaries due to fibrothecomas and a shortened life expectancy due to the Mulibrey heart disease $^{3}$ may prohibit this feature to become more evident. One patient was diagnosed with uterine endometrioid adenocarcinoma with a concomitant new thecoma 9 years after her one ovary had been removed because of a thecoma. This case could be explained by thecoma-related hyperestrogenism, a well-established risk factor of endometrial adenocarcinoma, or be coincidental. Wilms' tumor has been reported in about $4 \%$ of patients with Mulibrey nanism, ${ }^{2,21,22}$ but none were observed in our cohort.

In addition to Mulibrey nanism, ovarian sex cordstromal tumors are characteristic for several other inherited disorders. Ovarian fibrotic tumors at early age are a common finding in nevoid basal cell carcinoma syndrome (Gorlin syndrome), an autosomal-dominant inherited disorder caused by mutation in the human homologue of Drosophila patched gene $(P T C H)$ at $9 \mathrm{q} 22.3 .^{23}$ Sotos syndrome (cerebral gigantism), caused by mutations in the NSD1 gene, ${ }^{24}$ is another pleiotropic congenital syndrome were ovarian fibromas have earlier been described. ${ }^{25}$ Patients with Peutz-Jeghers syndrome, caused by germ-line inactivating mutations of the $S T K 11 / L K B 1$ 


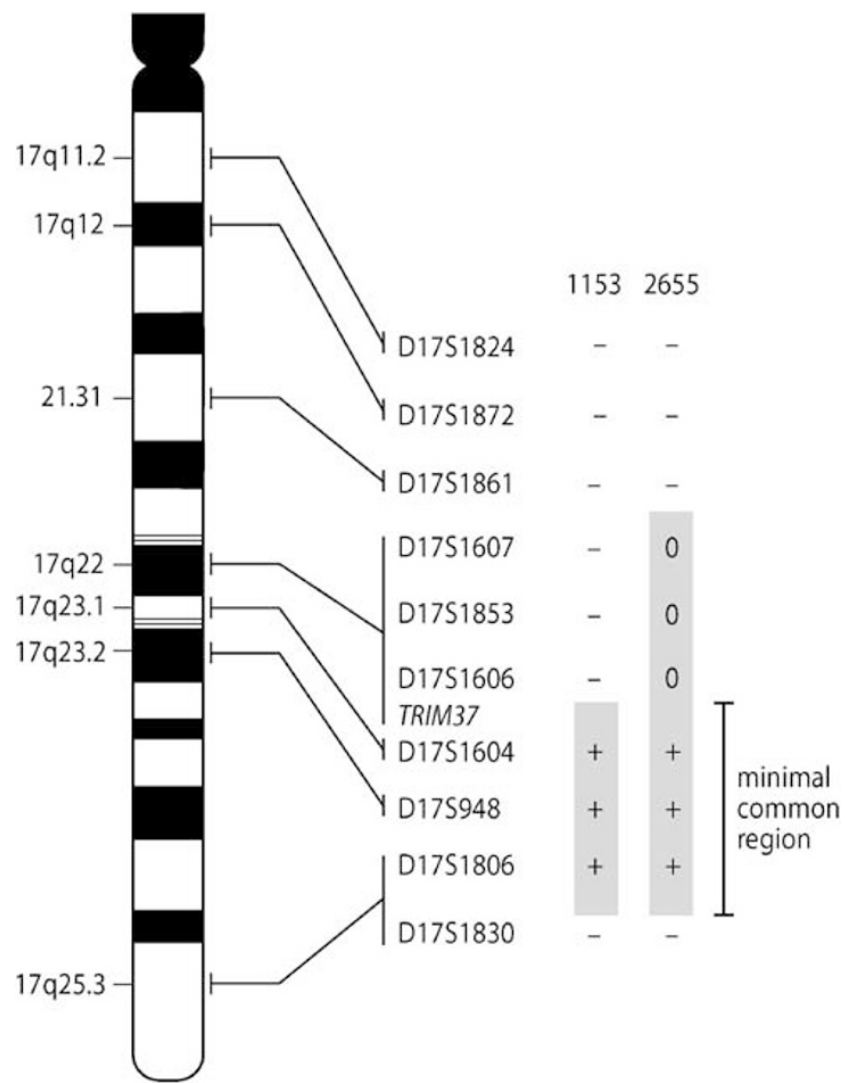

Figure 3 Deletion map of two sporadic fibrothecomas showing loss of heterozygosity at chromosome 17q. The genetic order of 10 microsatellite markers and TRIM37 are shown on the right side of the chromosome $17 \mathrm{q}$ and the corresponding loci are shown on the left side. +, LOH; -, informative with no loss; 0, not informative. Shaded area, potential deletions that include markers showing $\mathrm{LOH}$ and flanking noninformative markers.

gene at $19 \mathrm{p} 13.3,{ }^{26}$ are susceptible to sex cordstromal tumors with annular tubules. ${ }^{27}$

The genes responsible for development of sporadic fibrothecomas are unknown. Gain of chromosome 12 (trisomy or tetrasomy) is the most common and often the only genetic aberration detected by cytogenetic studies, including FISH in these tumors. ${ }^{28,29}$ A less frequent finding is monosomy of chromosome $22^{30}$ and recent studies also found imbalances in chromosomes 4, 9, 10 and 18 in this group of tumors. ${ }^{31,32}$ Allelic imbalance at 9q22.3 and 19p13.3 suggests possible involvement of PTCH and STK11 in sporadic fibrothecomas. ${ }^{33-35}$ However, no mutations of STK11 have been identified in sporadic sex cord-stromal tumors. ${ }^{34,35}$ To our knowledge, allelic imbalance involving chromosome 17 , site of TRIM37, has not been previously reported in fibrothecomas.

There are many examples of genes responsible for hereditary cancer syndromes being defective in sporadic tumors with similar morphology and location. One allele with loss-of-function mutation is in the germ line and the other one is inactivated by allele loss, mutation or epigenetic events like promoter hypermethylation or translational downregulation. The frequency of fibrothecomas in female patients with Mulibrey nanism prompted us to study TRIM37 gene and protein status in sporadic fibrothecomas. TRIM37 transcript was recovered from all freshly frozen tissue samples. In one tumor, a base pair change from $\mathrm{C} \rightarrow \mathrm{T}$ at nucleotide 398 was revealed, resulting in a substitution of valine for alanine. Although these belong to the same group of amino acids, the possibility for a biological function of the substitution cannot be ruled out. TRIM37 has several alternatively spliced variants, most of which predict nonfunctional protein products and may have a role in the regulation of TRIM37 expression. ${ }^{18}$ In sporadic fibrothecomas two splice variants were observed and verified by sequencing, deletion of exon 2 and exon 23. Previous studies have shown constitutional expression of TRIM37del23 in many tissues, and its function is unknown. ${ }^{18}$ TRIM37del2 has been earlier detected in testis. ${ }^{18}$ We found this variant in normal human ovary and most of the other normal tissues studied. However, as compared to normal ovary, in a proportion of fibrothecomas the relative amount of TRIM37del2 was significantly increased. This finding is especially interesting as deletion of exon 2 predicts a protein that lacks nearly the entire RING domain, which is needed for ubiquitin E3 ligase activity of TRIM37. ${ }^{9}$ The significance of TRIM37del2 in the pathogenesis of sporadic fibrothecomas remains to be studied.

In mouse, both TRIM37 gene and protein are expressed in a tissue-specific manner during ontogenesis. ${ }^{36,37}$ Also in adult human tissues the distribution of TRIM37 is confined to certain tissues and cells, which include ovary and testis, suggesting for TRIM37 a specific regulatory function rather than a more general role in cellular homeostasis. ${ }^{6,8,18}$ In normal human ovary, TRIM37 protein localized to the stromal cells, particularly the thecal cells surrounding the developing follicles, and smooth muscle cells of the vessels. Using this staining intensity as a reference, most sporadic fibrothecomas showed reduced or absent protein expression by immunohistochemistry. This was seen both in sparsely cellular fibromas and cellular thecomas, suggesting that it was not merely due to acquisition of less specialized phenotype. TRIM37 transcript was found in all sporadic fibrothecomas examined but $80 \%(20 / 25)$ of the tumors showed reduced or absent expression of TRIM37 protein, suggesting alterations at the translational level.

To further investigate the possible genetic and epigenetic events leading to the reduced expression of TRIM37 in sporadic fibrothecomas, we looked for $\mathrm{LOH}$ and $\mathrm{CpG}$ island promoter hypermethylation in these tumors. Of the sporadic fibrothecomas examined, $6 \%$ showed LOH with 3 microsatellite markers with a minimal common region containing the TRIM37 locus. Lack of allelic imbalance with 
markers distal to TRIM37 locus increases the significance of this observation. Methylation of TRIM37 promoter was present in $48 \%$ of the sporadic fibrothecomas examined, suggesting that this could be one mechanism of TRIM37 inactivation.

Sequences at $3^{\prime}$ UTR may also affect the localization of mRNA and antisense transcripts may regulate their sense counterparts. Interestingly, in testis transcripts with alternative $3^{\prime}$ UTR have been characterized suggesting that, indeed, TRIM37 may be regulated at translational level. ${ }^{18}$ In the complementary strand, partly overlapping the $3^{\prime}$ UTR of TRIM37 is the $3^{\prime}$ end of PPMIE, a gene belonging to the PP2C family. ${ }^{18}$ MicroRNAs negatively regulate translation by targeting mRNAs for cleavage or translational repression. ${ }^{38}$ TRIM37 $3^{\prime}$ UTR contains several putative miRNA targets. Further studies are needed to understand the possible mechanisms of translational downregulation of TRIM37 in sporadic fibrothecomas.

Our results show that inherited biallelic mutation of TRIM37 (Mulibrey nanism) predisposes to both mesenchymal and epithelial ovarian tumors. In sporadic fibrothecomas no TRIM37 mutations were found, but a proportion of tumors show increased expression of alternatively spliced TRIM37 variant lacking exon 2 and allelic loss at 17q22-25 including TRIM37 locus. Nearly half of the sporadic fibrothecomas showed evidence of $\mathrm{CpG}$ promoter methylation and TRIM37 protein was downregulated in the majority of the tumors, suggesting a role for TRIM37 in the pathogenesis of sporadic fibrothecomas.

\section{Acknowledgements}

We thank Gynel Arifdshan for excellent technical assistance, Kristiina Avela for help with the mutation analysis and Riikka Hämäläinen for helpful advice. This work was supported by grants from the Cancer Society of Finland, Foundation for the Finnish Cancer Institute, the Academy of Finland, the Folkhälsan Research Foundation, Helsinki University Central Hospital, the Finnish Foundation for Pediatric Research, Finska Läkaresällskapet and Medicinska understödsföreningen Liv och Hälsa.

\section{Conflict of interest}

None.

\section{References}

1 Perheentupa J, Autio S, Leisti S, et al. Mulibreynanism, an autosomal recessive syndrome with pericardial constriction. Lancet 1973;2:351-355.
2 Karlberg N, Jalanko H, Perheentupa J, et al. Mulibrey nanism: clinical features and diagnostic criteria. J Med Genet 2004;41:92-98.

3 Lipsanen-Nyman M, Perheentupa J, Rapola J, et al. Mulibrey heart disease: clinical manifestations, longterm course and results of pericardiectomy in a series of 49 patients born before 1985. Circulation 2003;107:2810-2815.

4 Karlberg N, Jalanko H, Kallijärvi J, et al. Insulin resistance syndrome in subjects with mutated RING finger protein TRIM37. Diabetes 2005;54: 3577-3581.

5 Avela K, Lipsanen-Nyman M, Perheentupa J, et al. Assignment of the Mulibrey Nanism gene to $17 q$ by linkage-disequilibrium analysis. Am J Hum Genet 1997;6:896-902.

6 Avela K, Lipsanen-Nyman M, Idänheimo N, et al. Gene encoding a new RING-B-box-Coiled-coil protein is mutated in Mulibrey nanism. Nat Genet 2000;25: 298-301.

7 Hämäläinen RH, Avela K, Lambert JA, et al. Novel mutations in the TRIM37 gene in Mulibrey nanism. Hum Mutat 2004;23:522.

8 Kallijärvi J, Avela K, Lipsanen-Nyman M, et al. The TRIM37 gene encodes a peroxisomal RING-B-boxCoiled-coil protein: classification of Mulibrey nanism as a new peroxisomal disorder. Am J Hum Genet 2002;70:1215-1228.

9 Kallijärvi J, Lahtinen U, Hämäläinen $\mathrm{R}$, et al. TRIM37 defective in Mulibrey nanism is a novel RING finger ubiquitin E3 ligase. Exp Cell Res 2005;308: 146-155.

10 Jagiello P, Hammans C, Wieczorek S, et al. A novel splice site mutation in the TRIM37 gene causes Mulibrey nanism in a Turkish family with phenotypic heterogeneity. Hum Mutat 2003;21:630-635.

11 Hämäläinen RH, Mowat D, Gabbett MT, et al. Wilms' tumor and novel TRIM37 mutations in an Australian patient with Mulibrey nanism. Clin Genet 2006;70: 473-479.

12 Doganc T, Yuksel Konuk BE, Alpan N, et al. A novel mutation in TRIM37 is associated with Mulibrey nanism in a Turkish boy. Clin Dysmorphol 2007;16:173-176.

13 Karlberg S, Tiitinen A, Lipsanen-Nyman M. Failure of sexual maturation in Mulibrey nanism. N Engl J Med 2004;351:2559-2560.

14 Tavassoli FA, Devilee P, (eds) Tumours of the Breast and Female Genital Organs. World Health Organization Classifications of Tumours. IAARC Press: Lyon, France, 2003.

15 Kononen J, Bubendorf L, Kallioniemi A, et al. Tissue microarrays for high-throughput molecular profiling of tumor specimens. Nat Med 1998;4:844-847.

16 Lassus H, Laitinen MP, Anttonen M, et al. Comparison of serous and mucinous ovarian carcinomas: distinct pattern of allelic loss at distal $8 \mathrm{p}$ and expression of transcription factor GATA-4. Lab Invest 2001;81: 517-526.

17 Canzian F, Salovaara R, Hemminki A, et al. Semiautomated assessment of loss of heterozygosity and replication error in tumors. Cancer Res 1996;56: 3331-3337.

18 Hämäläinen RH, Joensuu T, Kallijärvi J, et al. Characterization of the Mulibrey nanism-associated TRIM37 gene: transcription initiation, promoter region and alternative splicing. Gene 2006;366:180-188. 
19 Young RH, Scully RE. Ovarian sex cord-stromal tumors: recent advances and current status. Clin Obstet Gynaecol 1984;11:93-134.

20 Aboud E. A review of granulosa cell tumours and thecomas of the ovary. Arch Gynecol Obstet 1997;259:161-165.

21 Similä S, Timonen M, Heikkinen E. A case of Mulibrey nanism with associated Wilms' tumor. Clin Genet 1980;17:29-30.

22 Seemanova E, Bartsch O. Mulibrey nanism and Wilms tumor. Am J Med Genet 1999;85:76-78.

23 Hahn H, Wicking C, Zaphiropoulos PG, et al. Mutations of the human homolog of Drosophila patched in the nevoid basal cell carcinoma syndrome. Cell 1996;85:841-851.

24 Kurotaki N, Imaizumi K, Harada N, et al. Haploinsufficiency of NSD1 causes Sotos syndrome. Nat Genet 2002;30:365-366.

25 Chen CP, Yang YC, Lin SP, et al. Bilateral calcified ovarian fibromas in a patient with Sotos syndrome. Fertil Steril 2002;77:1285-1287.

26 Hemminki A, Markie D, Tomlinson I, et al. A serine/ threonine kinase gene defective in Peutz-Jeghers syndrome. Nature 1998;391:184-187.

27 Young RH, Welch WR, Dickersin GR, et al. Ovarian sex cord tumor with annular tubules: review of 74 cases including 27 with Peutz-Jeghers syndrome and four with adenoma malignum of the cervix. Cancer 1982;50:1384-1402.

28 Fletcher JA, Gibas Z, Donovan K, et al. Ovarian granulosa-stromal cell tumors are characterized by trisomy 12. Am J Pathol 1991;138:515-520.

29 Liang SB, Sonobe H, Taguchi T, et al. Tetrasomy 12 in ovarian tumors of thecoma-fibroma group: a fluorescence in situ hybridization analysis using paraffin sections. Pathol Int 2001;51:37-42.

30 Dal Cin P, Qi H, Pauwels P, et al. Monosomy 22 in a fibrothecoma. Cancer Genet Cytogenet 1997;99:129-131.

31 Micci F, Haugom L, Abeler VM, et al. Consistent numerical chromosome aberrations in thecofibromas of the ovary. Virchows Arch 2008;452:269-276.

32 Streblow RC, Dafferner AJ, Nelson M, et al. Imbalances of chromosomes 4, 9, and 12 are recurrent in the thecoma-fibroma group of ovarian stromal tumors. Cancer Genet Cytogenet 2007;178:135-140.

33 Tsuji T, Catasus L, Prat J. Is loss of heterozygosity at 9q22.3 (PTCH gene) and 19p13.3 (STK11 gene) involved in the pathogenesis of ovarian stromal tumors? Hum Pathol 2005;36:792-796.

34 Connolly DC, Katabuchi H, Cliby WA, et al. Somatic mutations in the STK11/LKB1 gene are uncommon in rare gynecological tumor types associated with PeutzJegher's syndrome. Am J Pathol 2000;156:339-345.

35 Kato N, Romero M, Catasus L, et al. The STK11/LKB1 Peutz-Jegher gene is not involved in the pathogenesis of sporadic sex cord-stromal tumors, although loss of heterozygosity at 19p13.3 indicates other gene alteration in these tumors. Hum Pathol 2004;35:1101-1104.

36 Lehesjoki AE, Reed VA, Mark Gardiner R, et al. Expression of MUL, a gene encoding a novel RBCC family ring-finger protein, in human and mouse embryogenesis. Mech Dev 2001;108:221-225.

37 Kallijärvi J, Hämäläinen R, Karlberg N, et al. Tissue expression of the Mulibrey nanism-associated Trim37 protein in embryonic and adult mouse tissues. Histochem Cell Biol 2006;126:325-334.

38 Bartel DP. MicroRNAs: genomics, biogenesis, mechanism, and function. Cell 2004;116:281-297. 